UCRL-ID-123065

\title{
Generalized Energy-Aperture Product Limit for Multi-Beam and Spotlight SARs
}

Thomas J. Karr

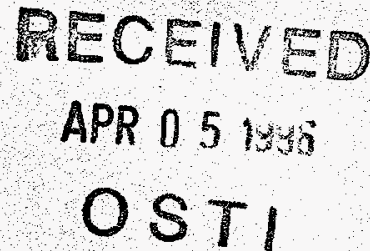

21 December 1995

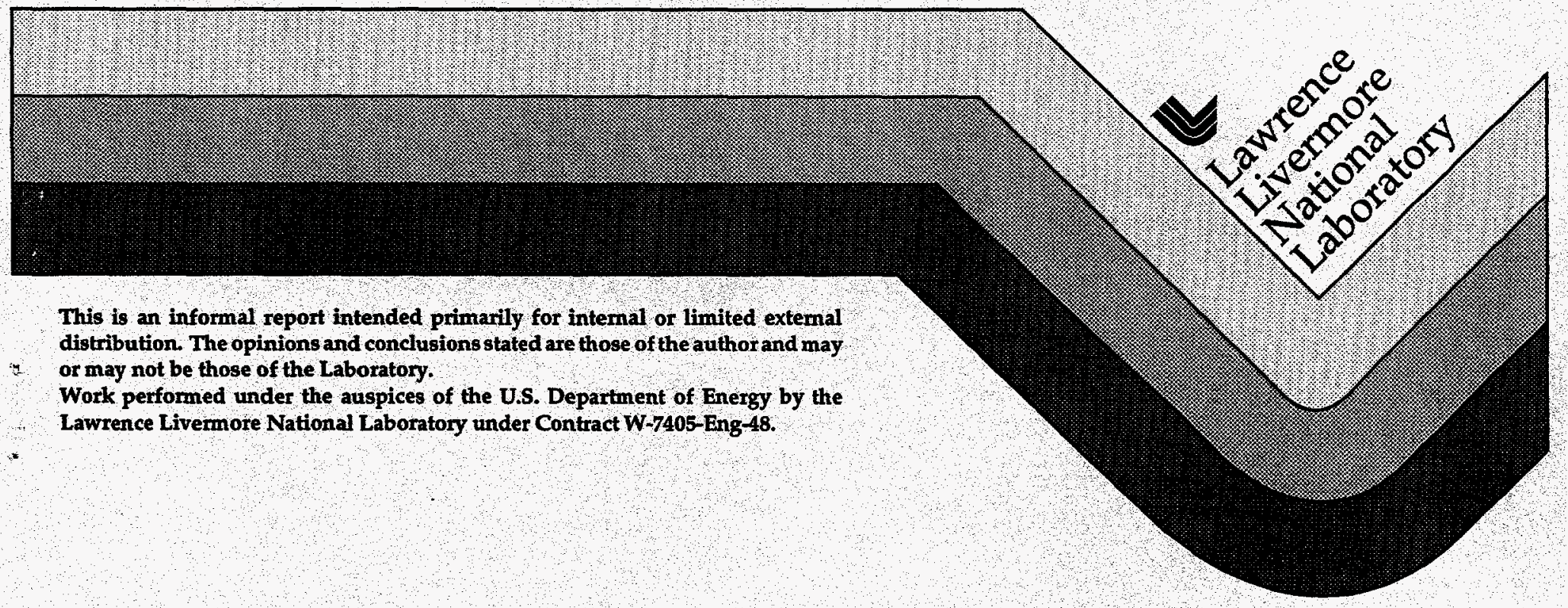




\section{DISCLAIMER}

This document was prepared as an account of work sponsored by an agency of the United States Government. Neither the United States Government nor the University of California nor any of their emplojees, makes any warranty, express or implied, or assumes any legal liability or responsibility for the accuracy, completeness, or usefulness of any information, apparatus, product, or process disclosed, or represents that its use would not infringe privately owned rights. Reference herein to any specific commercial products, process, or service by trade name, trademark, manufacturer, or otherwise, does not necessarily constitute or imply its endorsement, recommendation, or favoring by the United States Government or the University of Calffornia. The viewsand opinions of authors expressed herein do not necessarily state or reflect those of the United States Government or the University of California, and shall not be used for advertising or product endorsement purposes.

This report has been reproduced directly from the best avaliable copy.

Available to DOE and DOE contractors from the

Office of Scientific and Technical Information P.0. Box 62, Oak Ridge, TN 37831

Prices available from (615) 576-8401, FTS 626.8401

Available to the public from the National Technical Information Service

US. Department of Commerce 5285 Port Royal Rd. Springfield, VA 22161 


\title{
Generalized Energy-Aperture Product Limit for Multi-Beam and Spotlight SARs
}

\author{
Thomas J. Karr * \\ Lawrence Livermore National Laboratory, Livermore, CA 94550, USA
}

21 December 1995

\begin{abstract}
The SAR energy-aperture product limit is extended to multi-beam SARs, spotlight and moving spotlight SARs. This fundamental limit bounds the tradeoff between energy and antenna size. The kinematic relations between design variables such as platform speed, pulse repetition frequency, beam width and area rate are analyzed in a unified framework applicable to a wide variety of SARs including strip maps, spotlights, vernier arrays and multi-beam SARs, both scanning and swept-beam. Then the energy-aperture product limit is derived from the signal-to noise requirement and the kinematic constraints. The derivation clarifies the impact of multiple beams and spotlighting on SAR performance.
\end{abstract}

\footnotetext{
* Present address: LLNL, L-278, P.O. Box 808, Livermore CA 94550, tel. (510) 423-6270.
} 
We present a derivation of the SAR energy-aperture product limit generalized to multi-beam SARs, spotlight and moving spotlight SARs. This limit bounds the tradeoff between energy and antenna size. The derivation clarifies the impact of multiple beams and spotlighting on SAR performance.

\section{Kinematic Relationships}

We begin with a review of the basic SAR kinematics. The area rate of a single beam SAR, in strip-map, spotlight or moving spotlight mode, is given by

$$
\dot{A_{1}}=\delta R \cdot \tilde{V}
$$

where $\tilde{V}$ is the average speed of the beam along the direction of platform motion (average over time; the beam may step-stare or move in a non-uniform manner), and $\delta R$ is the cross-track width of the beam. The actual platform speed $V$ is related to the average beam speed by

$$
V=\zeta \tilde{V}
$$

where $\zeta$ is the spotlight factor.

The single-beam ground strip, of width $\delta R$ in the cross-track direction, is collected unambiguously if the pulse repetition frequency is below the limit

$$
P R F \leq \frac{c \sin \theta}{\left(K_{r}+1\right) \delta R \cos \psi}
$$

with $K_{r} \geq 1 . \theta$ is the squint angle on the ground and $\psi$ is the grazing angle 1 . Azimuth cells are collected to an acceptable ambiguity level if

$$
P R F \geq \frac{k_{a z R} V\left(K_{a z}+1\right) \sin \varphi}{L_{a z R}}
$$

with $K_{a z} \geq 1$ chosen to set the ambiguity level of the image. $L_{a z R}$ is the physical size of the receiver antenna in the azimuth direction, and the factor $k_{a z R}$ defines the useful edge of the beam $2,3,4$. The physical antenna range size for the transmitter is

1 We consistently use lowercase " $r$ " and "az" to denote parameters related to the physical range and azimuth, uppercase "SA" to denote parameters related to the synthetic aperture, and uppercase " $R$ " and " $T$ " to denote parameters related to the receiver and transmitter.

2 The significance of the " $\mathrm{k}$-factors" is that they define the effective edges of the beam in range, azimuth, or synthetic azimuth. For example, if the useful beam is defined to be all the beam between the $\mathrm{dB}$ points, and if the transmit aperture is uniformly illuminated, then $k_{a z T}=0.885$. The k-factors do not define the antenna gain. They do, however, influence several contributions 


$$
L_{r T}=\frac{k_{r T} \lambda R \sin \theta}{\delta R \sin \psi}
$$

where $\lambda$ is wavelength and $R$ is the range to the target area (at Doppler angle $\varphi$ ).

The effective area rate of a single-beam SAR also is given by

$$
\dot{A}_{1}=\frac{k_{a z T} \lambda R \delta R}{L_{a z T} \sin \theta} \cdot \frac{1}{T_{S A}}
$$

where $T_{S A}$ is the synthetic aperture integration time. The integration time is given in terms of the true azimuth resolution $\rho_{a z}$ and the synthetic aperture beamwidth factor $k_{S A}$ by

$$
T_{S A}=\frac{L_{S A}}{V \sin \varphi}=\frac{k_{S A} \lambda R}{2 p_{a z} V \sin \varphi}
$$

Combining (A), (F) and (G) and solving for $\zeta$ we get

$$
\zeta=\frac{L_{a z T} k_{S A} \sin \theta}{2 p_{a z} k_{a z T} \sin \varphi}
$$

This is the relationship between the spotlight factor, the effective azimuth size and the true azimuth resolution. Note that the true azimuth resolution may be different than the requirement, if the spotlight factor and antenna azimuth size are independently chosen or specified.

Combining the minimum and maximum pulse repetition frequencies of Equations (C) and (D), we solve for $V$ and the area rate:

$$
\begin{gathered}
V=\zeta \tilde{V} \leq \frac{c L_{a z R} \sin \theta}{k_{a z R} \delta R\left(K_{r}+1\right)\left(K_{a z}+1\right) \cos \psi \sin \varphi} \\
\dot{A}_{1} \leq \frac{2 c \rho_{a z}\left(L_{a z R} / L_{a z T}\right)\left(k_{a z T} / k_{a z R}\right)}{k_{S A}\left(K_{r}+1\right)\left(K_{a z}+1\right) \cos \psi} .
\end{gathered}
$$

to the total loss. For example, edge loss depends on just where the useful edge of the beam is defined to be.

3 We do not use "effective antenna sizes." The k-factors have meaning by themselves without reference to fictitious "effective sizes."

4 We allow for the possibility that the receiver size can be made be larger than the transmitter size by, for example, use of several receiver antennas to simultaneously collect the radar return at several points in space.

${ }^{5} k_{S A}$ may be $\geq 1$ to accommodate tapering of the synthetic aperture. 
The area rate is fixed by the imaging requirements for $\rho_{a z}, k_{S A}, k_{r} k_{a z}, K_{r}$ and $K_{a z}$. But any platform speed $V$ can be made consistent with the requirements, by an appropriate choice of the azimuth size $L_{a z T}$ (or equivalently the spotlight factor $\zeta$ and the single-beam strip width $\delta R$ (or equivalently the range size $L_{r T}$ ).

\section{Some Specific Cases}

The kinematic relations clarify the similarities and differences among various types of SAR. In the following we compare some typical SARs.

\section{Standard Strip Map}

A standard strip map by definition moves the beam at the same average speed as the platform, so $\zeta=1$. Therefore by Equation $(\mathrm{H})$ a strip map must have

$$
L_{a z T}=2 \rho_{a z} \frac{k_{a z T}}{k_{S A}} \frac{\sin \varphi}{\sin \theta}
$$

There are several other cases that make a "pseudo strip-map," typically by using $N$ azimuth beams each of which spotlights with $\zeta=N$ but which collectively scan at the platform speed; SCANSAR and VADO are examples of this. Such pseudo strip-maps do not satisfy Equation (K). But every single-beam SAR that truly sweeps area at the platform speed must satisfy Equation (K) ${ }^{6}$. It follows by Equation (J) that a true strip map beam may only have range width of

$$
\delta R \leq \delta R_{0}=\frac{2 c \rho_{a z}\left(L_{a z R} / L_{a z T}\right)\left(k_{a z T} / k_{a z R}\right)}{V k_{S A}\left(K_{r}+1\right)\left(K_{a z}+1\right) \cos \psi}
$$

If $\delta R_{O}$ is less than the desired map width, then spotlighting or moving spotlighting must be used.

\section{Spotlighted Strip Map}

A spotlighted strip map by definition has $\zeta>1$, i.e. thebeam moves slower than the platform 7 . It follows from Equation (I) that the single-beam strip width $\delta R$ can be larger than $\delta R_{0}$ :

$$
\delta R \leq \zeta \cdot \delta R_{0}
$$

6 Often, "strip map" is used to describe a collection where $\zeta \approx 1$ (but not necessarity $\zeta=1$ ). One example is a nadir-stabilized spaceborne SAR that pitches at $360 \%$ rev while collecting a map at broadside. In such cases the theory for the Spotlighted Strip Map with $\zeta>1$ should be used.

7 The beam may also be made to move faster than the platform. This could be the case, for example, if the map is not oriented parallel to the ground track. This normally would not be called "spotlighting," but the theory correctly includes it with $\zeta<1$. 
However, all single-beam SARs have the same area rate limit regardless of spotlighting:

$$
A_{1}=\tilde{V} \delta R \leq \frac{V}{\zeta} \cdot \zeta \delta R_{0}=V \delta R_{0}
$$

\section{Multiple Beams}

A typical multi-beam SAR has $N$ transmit beams operating in parallel, distinguished from each other in some way such as azimuth, elevation or frequency. Each beam moves at speed $\tilde{V}$. The combined $N$ beams, with or without spotlighting, collect at rate

$$
A_{N}=N A_{1}=N V \delta R_{0}
$$

\section{Vernier Array (VA)}

The VA SAR uses $N_{r}$ antennas to receive the return from each transmitter; there may also be $N_{t}$ multiple transmitters, distinguished from each other in some way such as azimuth or elevation angle or frequency. Since there are several simultaneous receivers, it is permissible to reduce the PRF below the ambiguity limit of a single receiver; the minimum PRF is given by Equation (D) with the appropriate $L_{a z R}>L_{a z T}$. Then by Equation (L) the VA SAR can have a larger strip width $\delta R_{V A}$, and therefore a larger collection area rate, than a conventional SAR with $L_{a z R}=L_{a z T}$ and strip width $\delta R_{O}$.

\section{Differential Offiset (DO)}

The DO SAR uses $N_{f}$ different transmit beams, each with a distinct frequency subband, imaging $N_{f}$ patches of target area (one subband to each patch). The beams may be spread out in azimuth, elevation, or both. Each beam spotlights with $\zeta=N_{f}$ over a strip of width $\delta R$ given by Equation (M), and collects with area rate given by Equation $(\mathrm{N})$. The combined area rate of the $N_{f}$ beams is

$$
\dot{A}=N_{f} \dot{A}_{1}=\zeta V \delta R_{0}=V \delta R
$$

Therefore the DO beams can collectively move at the platform speed $V$.

\section{Vernier Array Differential Offset (VADO)}

The vernier array and differential offset approaches can be combined into VADO. VADO uses $N_{f}$ different transmit beams, each of which is received by $N_{r}$ different receive antennas. The $N_{f}$ beams collectively move at the platform speed $\mathrm{V}$, and the area rate is given by Equation (P) with $\delta R_{0} \rightarrow \delta R_{V A}$. Since $\delta R_{V A}$ can be larger than $\delta R_{O}$. the VADO can collect at a higher rate than a non-VADO multi beam SAR with the same number of beams. 


\section{Derivation of the Energy-Aperture Product Limit}

We begin with the radar equation for the required average power:

$$
P_{\text {avg }}=\frac{4 \pi \lambda^{2} k T_{0} F L R^{4} \cos \psi}{\varepsilon_{T} \varepsilon_{R} A_{T} A_{R} \sigma_{n} \rho_{r} \rho_{a z} T_{S A}}
$$

where $T_{o}$ and $F$ are the receiver noise temperature and figure, $L$ is the total round-trip loss, $\sigma_{n}$ is the normalized backscattering cross-section, $\rho_{r}$ is the slantplane range resolution, $A_{T, R}$ is the transmitter/receiver antenna area, and $\varepsilon_{T, R}$ is the transmitter/receiver antenna efficiency. With the substitutions $A_{T}=L_{r T} L_{a z T}$, Equation (E) for $L_{r T}$, and $\rho_{r}^{G}=\rho_{r} / \cos \psi$ for the ground-plane resolution, the average power is

$$
P_{\text {avg }}=\frac{4 \pi \lambda k T_{0} F L R^{3} \delta R \sin \psi}{\varepsilon_{T} \varepsilon_{R} k_{r T} L_{a z T} A_{R} \sigma_{n} \rho_{r}^{G} \rho_{a z} T \sin \theta}
$$

Substituting Equation (G) for the synthetic aperture integration time gives

$$
P_{\text {avg }}=\frac{8 \pi V k T_{0} F L R^{2} \delta R \sin \psi \sin \varphi}{\varepsilon_{T} \varepsilon_{R} k_{r T} k_{S A} L_{a z T} A_{R} \sigma_{n} \rho_{r}^{G} \sin \theta}
$$

Eliminating $V$ by using Equation (I), the average power required is

$$
P_{a v g} \leq \frac{8 \pi c k T_{0} F L R^{2}\left(L_{a z R} / L_{a z T}\right) \tan \psi}{\left(K_{r}+1\right)\left(K_{a z}+1\right) \varepsilon_{T} \varepsilon_{R} k_{r T} k_{a z R} k_{S A} A_{R} \sigma_{n} \rho_{r}^{G}}
$$

This is a general result. It does not depend directly on the spotlight factor but it does depend on the range size and azimuth size of the receiver, both of which may be chosen at will by the SAR designer.

The time required for the single-beam SAR to map the area is

$$
T_{\text {equiv }}=\frac{A_{1}}{\dot{A}_{1}} \geq \frac{k_{S A}\left(K_{r}+1\right)\left(K_{a z}+1\right) A_{1} \cos \psi}{2 c \rho_{a z}\left(L_{a z R} / L_{a z T}\right)\left(k_{a z T} / k_{a z R}\right)} .
$$

The total energy required for the single-beam SAR map is

$$
E_{1}=P_{\text {avg }} T_{\text {equiv }}=\frac{4 \pi A_{1} k T_{0} F L R^{2} \sin \psi}{\varepsilon_{T} \varepsilon_{R} k_{r T} k_{a z T} A_{R} \sigma_{n} \rho_{r}^{G} \rho_{a z}} .
$$

Therefore the required energy-aperture product for the single-beam SAR map is 


$$
\Phi_{1}=E_{1} A_{R}=\frac{4 \pi A_{1} k T_{0} F L R^{2} \sin \psi}{\varepsilon_{T} \varepsilon_{R} k_{r T} k_{a z T} \sigma_{n} \rho_{r}^{G} \rho_{a z}}
$$

Note that the only influence the receiver has on $\Phi_{1}$ is through the antenna efficiency $\varepsilon_{R}$. and noise figure $F$. Also note that the energy-aperture product depends only indirectly on squint through the range.

A single-beam SAR can always be designed to image the required area at any specified platform speed $V$ by choosing $L_{a z T}$ satisfying Equation (I) 8 . Consequently, a single-beam SAR can always be designed to meet the imaging requirements; it would have energy-aperture product $\Phi_{1}$ of Equation (W).

The number of SAR beams is entirely a matter of design choice. If the requirement is to image a total area $\mathrm{A}$ with a total strip width $\Delta R$, then the designer may choose to collect the entire strip width and area with a single SAR beam ( $\delta R=\Delta R$ in all the formulae above). Alternatively the designer may choose to make the single-beam collection area $A_{1}$ less than $A$ in order to meet some other design goal (e.g. fit into a given launcher envelope, mass budget, or cost). In that case the area $A$ would be divided into several parts, and several SAR beams would be used to collect it. The number $N$ of SAR beams would be

$$
N=\frac{A}{A_{1}} .
$$

The designer could use multiple elevation beams by choosing to make $\delta R \leq \Delta R$, in which case the relation between the single-beam area imaged and total area imaged is

$$
A=\frac{\Delta R}{\delta R} A_{1}
$$

or he could choose to make multiple azimuth beams, in which case the total collection time is reduced to

$$
T_{\text {true }}=\frac{A}{N \dot{A}_{1}}=\frac{A_{1}}{\dot{A}_{1}} \quad ;
$$

or he could choose to use a combination of elevation and azimuth beams. But $N$ is always under the control of the designer and is not fixed by the imaging

8 A single-beam SAR would theoretically be possible; we do not claim in all cases it would be attractive. For example, the resulting spotlight factor given by Equation (H) may not be acceptable to the designer due to its impact on the spacecraft. But that would be for reasons other than the basic imaging requirements of resolution, target area and ambiguity rejection. 
requirements. Each of the $N$ beams has the energy-aperture product limit $\Phi_{1}$, Equation (W).

In all cases the required product of transmitted energy and receiver aperture of the multi-beam SAR, for any number of beams, is given by replacing $A_{1}$ with $A$ :

$$
\Phi=N \Phi_{1}=\frac{4 \pi A k T_{0} F L R^{2} \sin \psi}{\varepsilon_{T} \varepsilon_{R} k_{r r} k_{a z} \sigma_{n} \rho_{r}^{G} \rho_{a z}} .
$$

The imaging requirements determine $\Phi$ by Equation (AA); imaging requirements plus the design choices of $\zeta$ and $\delta R$ (or equivalent parameters such as $L_{r T}$ and $L_{a z T}$ ) determine all the other SAR parameters.

\section{Discussion}

The energy-aperture product, Equation (AA), is our principal result. The energy-aperture product limit Equation (AA) applies to all SAR implementations regardless of the number of beams (or any other design choice).

The area rate does not put any constraint on the number of SAR beams. The designer may simultaneously choose to make each beam of his SAR collect at an area rate $A_{1}$ and make his whole system collect at a larger rate $A$, in which case Equation (X) gives the number of SAR beams chosen by the designer (not demanded by imaging requirements). In every case the full-system energy aperture product is given by using the full imaging area $A$ in Equation (AA) above. The energy-aperture product should never be multiplied by the number of beams as it would correspond to an image area of $N \cdot A$.

\section{Acknowledgment}

The author is grateful to Jordin Kare for many stimulating discussions on this subject, and especially for his critical reading of the draft. This work was supported in part by the US Air Force. 